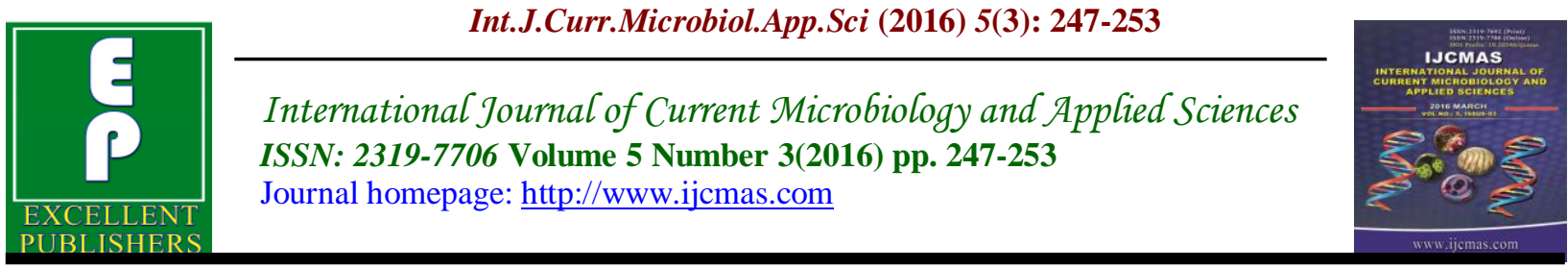

Original Research Article

http://dx.doi.org/10.20546/ijcmas.2016.503.030

\title{
Isolation of Pseudomonas aeruginosa from various Clinical Isolates and it Antimicrobial Resistance Pattern in a Tertiary Care Hospital
}

\author{
Saroj Golia, Suhani, S. Manasa* and Jyoti \\ Institute-Dr.B.R.Ambedkar Medical College, K.G.Halli, Bengaluru, India \\ *Corresponding author
}

\begin{abstract}
A B S T R A C T
Keywords

P. aeruginosa,

Antimicrobial

resistance

pattern,

Clinical isolates.

Article Info

Accepted:

15 February 2016

Available Online:

10, March 2016

Pseudomonas aeruginosa is an opportunistic human pathogen and is the leading cause of nosocomial infections especially in immune compromised patients. In recent years, a considerable increase in the prevalence of multidrug resistance (MDR) $P$. aeruginosa has been noticed with high morbidity and mortality, hence requiring antibiotic susceptibility testing on a regular as well as a periodic basis. The present study was undertaken to determine the antibiogram of $P$. aeruginosa and its frequency of occurence from various clinical samples. A study was undertaken with 120 samples which were taken from patients of Dr. B. R. Ambedkar Medical College and hospital. The study was carried out in the Department of Microbiology, Dr. B.R.A.M.C, K. G. Halli, Bengaluru for a period of 9 months from July 2014- March 2015.A total of 120 clinically significant $P$. aeruginosa isolates were collected from different clinical samples, and processed using conventional microbiological methods. The strains were cultured and identified by standard microbiological techniques and Kirby- Bauer disc diffusion antibiotic susceptibility testing was done for each. Majority of isolates of $P$. aeruginosa $(102 / 120,85 \%)$ were obtained from specimens of pus, sputum, ear discharge and tracheal aspirates. Among 112 isolated pathogens 35(31.25\%) showed resistance to aminoglycosides, $30(26.78 \%)$ to cephotaxime. Resistance rates to Piperacilin/tazobactam, ceftazidime, ofloxacin varied from $10-15$ (8.92\% to $13.39 \%) .10 / 112(8.92 \%)$ isolates were multi-drug resistant. All strains were found to be sensitive to imipenam, colistin $(100 \%)$. The results confirmed the occurrence of drug resistant strains of $P$. aeruginosa. Imipenem, colistin, ceftazidime, pipperacillin-tazobactem and cefipime were found to be the most effective antimicrobial drugs. It therefore calls for a very judicious, rational treatment regimens prescription by the physicians to limit the further spread of antimicrobial resistance among the $P$. aeruginosa strains.
\end{abstract}

\section{Introduction}

Pseudomonas aeruginosa is a nonfermentative, aerobic, motile, gram negative bacilli that belongs to the family, pseudomonadaceae. It was first isolated from green pus in 1882. More than half of all clinical isolates produce the blue-green 
pigment pyocyanin. Being an opportunistic human pathogen, it is the leading cause of nosocomial infections, especially among patients who are admitted to intensive care units. (ICU).It can survive with low levels of nutrients and grow in temperature raning from $4-42^{\circ} \mathrm{c}$. These characteristics allow it to attach itself and survive on medical equipment and on other hospital surfaces, which favours the beginning of infections in immune compromised patients(1).

According to data from the US Centers for Disease Control and Prevention and the National Nosocomial Infection Surveillance System, $P$. aeruginosa is the second most common cause of nosocomial pneumonia (17\%), the third most common cause of urinary tract infection (7\%), the fourth most common cause of surgical site infection $(8 \%)$, the seventh most frequently isolated pathogen from the bloodstream $(2 \%)$ and the fifth most common isolate $(9 \%)$ overall from all sites(2).

Antimicrobial agents have been the only easily and widely used therapeutic option available to counter the infections caused by diverse microbial agents. However, microbial populations have developed various strategies to overcome these antimicrobial agents - a major contributing factor in the development of anti-microbial resistance world-wide. The development of resistance to all available antibiotics in some organisms may preclude the effectiveness of any antibiotic regimen(3).

Mechanisms that cause antimicrobial drug resistance and multi-drug resistance in $P$. aeruginosa are due to acquisition of resistance genes (e.g those encoding betalactamase (3) and amino-glycoside modifying enzymes (7) via horizontal gene transfer and mutation of chromosomal genes (target site, efflux mutations) are the target of the fluoroquinolones particularly ciprofloxacin (3). Biofilm formation in $P$. aeruginosa, particularly in the case of pulmonary infections in patients with cystic fibrosis, contribute to its resistance to antimicrobial agents (3). MDR $P$. aeruginosa elaborates inactivating enzymes that make beta-lactams and carbapenems ineffective, such as extended spectrum beta lactamases (ESBLs) and metallo- $\beta$ lactamases (MBLs) (4). ESBL-producing $P$. aeruginosa was first detected in Western Europe in the mid-1980s, and MBLproducing $P$. aeruginosa was first reported from Japan in 1991. They have rapidly spread over different parts of world since then (4).

MDR $P$. aeruginosa phenotype is defined as a bacterium which is resistant to antimicrobial agents which are included in three or more anti-Pseudomonal anti-microbial classes (carbapenems, fluoroquinolones, penicillins /cephalosporins and aminoglycosides (ㄴ) .

With an increase in the number of multidrug resistant (MDR) strains of $P$. aeruginosa the availability of therapeutic options has been severely limited. This study was therefore designed to find out the current antimicrobial susceptibility patterns of $P$. aeruginosa strains in a centrally located urban tertiary care hospital at D.R.B.R.A.M.C.

\section{Materials and Methods}

\section{Setting}

The study was carried out in the Department of Microbiology, Dr. B.R.A.M.C, K.G.Halli, Bengaluru for a period of 9 months from July 2014- March 2015.A total of 120 clinically significant $P$. aeruginosa isolates were collected from different clinical samples and processed using conventional microbiological methods. 


\section{Laboratory Identification of Isolates}

The samples were cultured on Blood Agar and Mac Conkey's Agar, and the plates were incubated overnight at $37^{\circ} \mathrm{C}$. P. aeruginosa was identified by its colony characteristics, pigment production, grape like odour, oxidase positivity, motility, gram staining (as gram negative bacilli), ability of reducing nitrates to nitrites, nonfermentative character, along with its ability to decarboxylate arginine, liquefy gelatin and to grow at $42^{\circ} \mathrm{C}$ microbiological techniques (5).

\section{Antibiotic Susceptibility Testing}

Antibiotic sensitivity patterns of these isolates were studied by using Kirby Bauer Disc Diffusion method on Mueller -Hinton agar, by following CLSI 2014 Guidelines (6), by using Hi-media antibiotic discs. Antibiotics which were tested include piperacillin $(100 \mu \mathrm{g})$, ceftazidime $(30 \mu \mathrm{g})$ gentamicin (10mcg), amikacin (30 mcg), piperacillin + tazobactam $(100 / 10 \mathrm{mcg})$, imipenem (10mcg), ofloxacin $(05 \mathrm{mcg})$, cephotaxime (30mcg), cefoperazone (30mcg), cefipime $(30 \mathrm{mcg})$ and colistin (10mcg). Pseudomonas aeruginosa ATCC 27853 strain was used for quality control in the study. In our work, MDR $P$. aeruginosa was detected as a bacterium which was resistant to three or more anti-Pseudomonal anti-microbial classes (piperacillin + tazobactam, ofloxacin, cephotaxime and gentamicin) (4).

\section{Results and Discussion}

\section{Patients and Specimens Data}

120 strains of $P$. aeruginosa were isolated and identified by standard microbiological procedures, out of a total of 500 clinical specimens investigated. The rate of isolation of $P$. aeruginosa was $24 \%$. Of these 120 strains of $P$. aeruginosa, $80(66.6 \%)$ were from males and $40(33.3 \%)$ were from females. Most of them belonged to the age group 41-60 years $(40,33.3 \%)$, followed by patients of $>60$ years of age $(35,29.16 \%)$ as shown in Table 1.

Wound/pus, sputum and tracheal aspirates $(102 / 120,85 \%)$ were the predominant sources of specimens of $P$. aeruginosa.

Antibiogram results have been described in detail in Table 3 and they demonstrated among 112 isolated pathogens 35(31.25\%) showed resistance to aminoglycosides, $30(26.78 \%)$ to cephotaxime. Resistance rates to Piperacilin/ tazobactam, ceftazidime, ofloxacin varied from $10-15(8.92 \%$ to $13.39 \%) .10 / 112(8.92 \%)$ isolates were multidrug resistant.

All strains were found to be sensitive to imipenam, colistin (100\%).

In this study, a total of 120 isolates of $P$. aeruginosa were isolated and identified from various clinical sources, from the hospitalized patients and their antimicrobial susceptibility patterns were determined. Most of them belonged to older age group $41-60$ years $(40,33.3 \%)$, followed by patients of $>60$ years of age (35, $29.16 \%$ ). This could be explained as due to decreased immunity, prolonged hospitalization and other associated comorbidities in these age groups.

A study done in Kathmandu, Nepal showed a similar result (3), whereas according to a study done in Ahmadabad, India (8) shown $(29,29.00 \%)$ of patients were aged between 31-45 years. Sex-wise, male patients 80 $(66.6 \%)$ constituted a larger group in our study, simillarly Ahmed et al.,(9) reported an increased incidence in male sex $(77.7 \%)$ as well as a higher prevalence rate among elderly $\quad 61-80$ years $(43.92 \%)$. 
Table.1 Age and Gender Wise Distribution of Clinical Isolates of Pseudomonas aeruginosa

\begin{tabular}{|l|l|l|l|}
\hline Age group (years ) & Male (no.) & Female (no.) & Total (no.)\% \\
\hline$<20$ & 10 & 10 & $20(16.6 \%)$ \\
\hline $21-40$ & 15 & 10 & $25(20.8 \%)$ \\
\hline $41-60$ & 25 & 15 & $40(33.3 \%)$ \\
\hline$>60$ & 30 & 05 & $35(29.16 \%)$ \\
\hline Total & 80 & 40 & $120(100 \%)$ \\
\hline
\end{tabular}

Table.2 Distribution of Specimens of Pseudomonas aeruginosa Clinical Isolates

\begin{tabular}{|l|l|l|}
\hline Source of Specimen & Number & Percentage (\%) \\
\hline Pus / wound & 67 & $55.83 \%$ \\
\hline Sputum & 25 & $20.83 \%$ \\
\hline Urine & 06 & $5 \%$ \\
\hline Tracheal aspirate & 10 & $8.33 \%$ \\
\hline Ear discharge & 10 & $8.33 \%$ \\
\hline High Vaginal Swab & 02 & $1.66 \%$ \\
\hline Total & 120 & 100.00 \\
\hline
\end{tabular}

Table.3 Antimicrobial Susceptibility Patterns of Pseudomonas aeruginosa Clinical Isolates

\begin{tabular}{|l|l|l|}
\hline Antibiotic & Sensitive no.(\%) & Resistant no. (\%) \\
\hline Amikacin & $97(86.6 \%)$ & $15(13.3 \%)$ \\
\hline Gentamicin & $92(82.14 \%)$ & $20(17.85 \%)$ \\
\hline Piperacillin-tazobactam & $102(91.07 \%)$ & $10(8.92 \%)$ \\
\hline Piperacillin & $100(89.28 \%)$ & $12(10.71 \%)$ \\
\hline Ceftazidime & $102(91.07 \%)$ & $10(8.92 \%)$ \\
\hline Cephotaxime & $82(73.21 \%)$ & $30(26.78 \%)$ \\
\hline Cefipime & $107(95.5 \%)$ & $05(4.46 \%)$ \\
\hline Ofloxacin & $102(91.07 \%)$ & $10(8.92 \%)$ \\
\hline Imepenam & $112(100 \%)$ & 0 \\
\hline Colistin & $112(100 \%)$ & 0 \\
\hline
\end{tabular}

The distribution of specimens of $P$. aeruginosa may vary with each hospital as each hospital facility has a different environment associated with it. More than $80 \%$ of the $P$. aeruginosa isolates were obtained from wound / pus, sputum, urine and tracheal aspirates. Similar results had been obtained in different studies in India reported by Chander et al. (3), Mohanasoundaram (9) and Arora et al. (10) respectively.
Increasing resistance to different antipseudomonal drugs particularly among hospital strains, has been reported worldwide (19-20) and this is a serious therapeutic problem in the management of disease due to these organisms. Among the beta-lactams, $P$. aeruginosa showed highest resistance to cephotaxime (26.78\%). However, it was more sensitive to other beta-lactams i.e., piperacillin+ tazobactam, ceftazidime, cefipime and imipenem sensitive (91.07\%, 
$91.07 \%, 95.5 \%$ and $100 \%$ respectively) has been described. Hence ceftazidime and piperacillin+ tazobactam can be conviently used as first line drugs keeping imipenem as reseve drug for resistant cases

One striking feature in this study was that all the $P$. aeruginosa isolates were found to be sensitive to imipenem and Colistin. This may be due to the restricted use of imipenem in this hospital. This is consistent with a report published in 2002 in Mangalore, India (11) but other studies have showed varying degrees of resistance to imipenem in recent years $(09,10,12,13)$.

Cefipime(95.5\% sensitive), followed by ceftazidime, piperacillin+ tazobactam (both $91.07 \%$ sensitive) proved to be the most effective drugs for routine use among the $P$. aeruginosa strains investigated in this study. It has to be noted, that according to Srinivasan et al., $P$. aeruginosa was resistant to beta lactams viz. cephalothin, carbeniciilin, ceftazidime (100\%), and cephalexin (98\%) respectively (14). According to the study of Saha et al., it is most sensitive to beta lactams - imipenem (98.72\%), followed by aztreonam $(33.44 \%)$ and ceftazidime $(38.32 \%)(\underline{18})$.

Studies done by Kaushik et al. (15), Singh et al. (16), Taneja et al. (17) which were done in Indian context, showed resistance of Pseudomonas spp. in the range of 13.9 $90 \%$ to amikacin, in the range of $4-90 \%$ to ceftazidime, in the range of $50-77.7 \%$ to gentamicin and in the range of $41-95.1 \%$ to ciprofloxacin, which reflected high resistance profile of this nosocomial pathogen.

In the present study, MDR rate (resistance to three or more of anti Pseudomonal antimicrobials i.e (piperacillin + tazobactam, ofloxacin, cephotaxime and gentamicin) was determined to be low 10/112(8.92\%). A study done by Unan et al., (19) in Turkey reported rates of MDR, which were as high as $60 \%$, whereas study done by Sabir et al., in Pakistan detected lower rates of MDR (22.08\%) (20). Combination treatments are generally recommended for suspected Pseudomonas infections. It has been reported that the choice of a carbapenem, cefepime, or piperacillin+ tazobactam, in combination with amikacin or tobramycin, in current times, appears to provide the widest potential antimicrobial activity against MDR $P$. aeruginosa (18).

Rigorous monitoring for MDR among Pseudomonas isolates is very important, because outbreaks caused by strains which are resistant to potentially useful agents, including carbapenems, have been reported elsewhere $(09,10,12,13)$.

In conclusion, the present study concluded with high percentage of sensitive drugs and lower percentage of drug resistant strains. Though low, MDR strains were isolated which calls for rationale and judicious use of antibiotics. Hence in conclusion, confined usage of 'selected antibiotic' with effective application of infection control policies for each institution, would help to combat the rapid emergence of MDR $P$. aeruginosa. Regular anti-microbial susceptibility monitoring is essential which helps and guides the physicians to prescribe the right combinations of anti-microbials to limit and prevent the emergence of multi-drug resistant strains of $P$. aeruginosa.

\section{References}

Ahmed, S.M., Jakribettu, R.P., Kottakutty, S., Arya, B., Shakir, V.P.A. 2012. An emerging multi-drug resistant pathogen in a tertiary care centre in North Kerala. Annals. Biol. Res., 3(6): 2794-99. 
Al-Kabsi, A.M., Yusof, M.Y.B.M., Sekaran, S.D. 2011. Antimicrobial resistance pattern of clinical isolates of Pseudomonas aeruginosa in the University of Malaya Medical Center, Malaysia. Afr. J. Microbiol. Res., 5(29): 5266-72.

Arora, D., Jindal, N., Kumar, R., Romit. 2011. Emerging antibiotic resistance in Pseudomonas aeruginosa. Int. J. Pharm. Sci., 3(2): 82-4.

Chander Anil, Raza Mohammad Shahid. 2013. Antimicrobial susceptibility patterns of pseudomonas aeruginosa clinical isolates at a tertiary care hospital in Kathmandu, Nepal. Asian J. Pharm. Clin. Res., Vol. 6, Suppl. 3, 235-238.

Clinical and Laboratory Standards Institute (CLSI). 2014. Performance standards for antimicrobial susceptibility testing; Twenty fourth Informational supplement. M100-S24.

Collee, J.G., Fraser, A.G., Marmion, B.P., Simmons, A. 1996. Mackie and McCartney Practical Medical Microbiology. 14th ed. Edinburgh: Churchill Livingstone. 1996.

Indu Biswal, Balvinder Singh Arora, Dimple Kasana, Neetushree. 2014. Incidence of multidrug resistant Pseudomonas Aeruginosa isolated from Burn Patients and Environment of Teaching Institution. J. clin. Diag. Res. JCDR, $8(5)$.

Javiya, J.A., Ghatak, S.B., Patel, K.R., Patel, J.A. Antibiotic susceptibility patterns of Pseudomonas aeruginosa at a tertiary care hospital in Gujarat, India. Ind. J. Pharmacol., 40(5): 230-34.

Kaushik, R., Kumar, S., Sharma, R., Lal, P. 2001. Bacteriology of burn wounds the first three years in a new burn unit at the medical College, Chandigarh. Burns., 27: 595-7.
Mohanasoundaram, K.M. 2011. The antibiotic resistance pattern in the clinical isolates of Pseudomonas aeruginosa in a tertairy care hospital; 2008-2010 (A 3 year study). J. Clin. Diagn. Res., 5(3): 491-94.

National Nosocomial Infections Surveillance (NNIS) system report: data summary from January 1992 through June 2003, issued August 2003. http://www.cdc.gov/ncidod/ dhqp/pdf (15 January 2009, date last accessed).

Rajat, R.M., Ninama, G.L., Mistry, K., Parmar, R., Patel, K., Vegad, M.M. 2012. Antibitic resistance pattern in Pseudomonas aeruginos species isolated at a tertiary care hospital, Ahmadabad. Nat. J. Med. Res., 2(2): 156-59.

Sabir, R., Alvi, S.F.D., Fawwad, A. 2013. Antimicrobial susceptibility pattern of aerobic microbial isolates in a clinical laboratory in Karachi- Pakistan. Pak. J. Med. Sci., 29(3): 851-5.

Saha, S.K., Muazzam, N., Begum, S.A., Chowdhury, A., Islam, M.S., Parveen, R. 2011. Study on time-related changes in aerobic bacterial pattern of burn wound infection. Faridpur Med. Coll. J., 6(1): 41-5.

Shenoy, S., Baliga, S., Saldanha, D.R., Prashanth, H.V. 2002. Antibiotic sensitivity patterns of Pseudomonas aeruginosa strains isolated from various clinical specimens. Ind. $J$. Med. Sci., 56(9): 427-30.

Singh, N.P., Goyal, V., Manchanda, V., Das, S., Kaur, I., Talwar, V. 2003. Changing trends in bacteriology of burns in the burns unit, Delhi, India. Burns., 29: 129-32.

Srinivasan, S., Vartak, A.M., Patil, A., Saldanha, J. 2009. Bacteriology of burn wound at the Baba Jerbai Wadia hospital for children, Mumbai- A 13 year study. Ind. J. Plastic Surg., 42(2): 
213-8.

Stover, C.K., Pham, X.Q., Erwin, A.L, et al. 2000. Complete genome sequence of Pseudomonas aeruginosa PAO1, an opportunistic pathogen. Nature, 406: 959-64.

Taneja, N., Emmanuel, R., Chari, P.S., Sharma, M. 2004. A prospective study of hospital-acquired infections in burn patients at a tertiary care referral centre in North India. Burns., 30: 6659.

Unan, D., Gnseren, F. 2000. The resistance of $P$. aeruginosa strains isolated from nosocomial infections against various antibiotics. Mikrobiyol Bult., 34: 25560.

\section{How to cite this article:}

Saroj Golia, Suhani, Manasa S. and Jyoti. 2016. Isolation of Pseudomonas aeruginosa from various Clinical Isolates and it Antimicrobial Resistance Pattern in a Tertiary Care Hospital. Int.J.Curr.Microbiol.App.Sci. 5(3): 247-253. doi: http://dx.doi.org/10.20546/ijcmas.2016.503.030 